\title{
Cyanobacteria, microcystins and cylindrospermopsin in public drinking supply reservoirs of Brazil
}

\author{
MARIA DO CARMO BITTENCOURT-OLIVEIRA ${ }^{1,2,3}$, VIVIANE PICCIN-SANTOS ${ }^{1,3,4}$, \\ ARIADNE N. MOURA ${ }^{2}$, NÍSIA K.C. ARAGÃO-TAVARES ${ }^{2}$ and MICHELINE K. CORDEIRO-ARAÚJO ${ }^{1,2}$ \\ ${ }^{1}$ Departamento de Ciências Biológicas, Escola Superior de Agricultura Luiz de Queiroz, \\ Universidade de São Paulo, Av. Pádua Dias, 11, 13418-900 Piracicaba, SP, Brasil \\ ${ }^{2}$ Programa de Pós-Graduação em Botânica, Universidade Federal Rural de Pernambuco, \\ Rua D. Manoel de Medeiros, s/n, Dois Irmãos, 52171-030 Recife, PE, Brasil \\ ${ }^{3}$ Programa de Pós-Graduação em Ciências Biológicas, Universidade Estadual Paulista, UNESP, \\ Av. 24-A, 1515, 13506-900 Rio Claro, SP, Brasil \\ ${ }^{4}$ Universidade Estadual de Campinas, Centro Pluridisciplinar de Pesquisas Químicas, \\ Biológicas e Agrícolas, Rua Alexandre Caselatto, 999, 13081-970 Paulínia, SP, Brasil \\ Manuscript received on September 10, 2012; accepted for publication on May 8, 2013
}

\begin{abstract}
Brazil has a history of blooms and contamination of freshwater systems by cyanobacterial toxins. The monitoring relevance of toxins from cyanobacteria in reservoirs for public supply is notorious given its high toxicity to mammals, included humans beings. The most recurrent toxins in Brazilian water bodies are microcystins (MC). However, the recent record of cylindrospermopsin (CYN) in northeastern Brazil, Pernambuco state, alerts us to the possibility that this could be escalating. This study reports occurrence of $\mathrm{MC}$ and CYN, quantified with ELISA, in 10 reservoirs, devoted to public drinking supply in northeastern Brazil. The composition and quantification of the cyanobacteria community associated with these water bodies is also presented. From 23 samples investigated for the presence of MC, and CYN, 22 and 8 out were positive, respectively. Considering the similarity of the cyanobacteria communities found in reservoirs from Pernambuco, including toxin-producing species associated to MC and CYN, we suggest that geographic spreading can be favored by these factors. These issues emphasize the need for increased monitoring of $\mathrm{MC}$ and CYN in drinking supply reservoirs in Brazil.
\end{abstract}

Key words: bloom, harmful algae, monitoring, toxin, water quality.

\section{INTRODUCTION}

Toxins from cyanobacteria are known to be harmful agents to human and animal health (Carmichael 1994, Jochimsen et al. 1998, Soares et al. 2006). Besides the high poisoning potential of these toxins, chronic effects in human populations due to long-term

Correspondence to: Maria do Carmo Bittencourt-Oliveira E-mail: mbitt@usp.br exposure to hepatic and neurotoxins have also been reported (Suganuma et al. 1988, Falconer and Buckley 1989, Falconer 1991, 1996, Nishiwaki-Matsushima et al. 1992, Carmichael 1994, Ito et al. 1997, Ding et al. 1999, Cox et al. 2003, Clark et al. 2007).

Microcystins (MC) are hepatotoxins produced by some species of the Microcystis, Planktothrix and Anabaena/Dolichospermum genera, among others, 
which act by inflicting damage to cells from the liver and other organs (Soares et al. 2007), possibly leading the organisms to death by hemorrhagic shock (Mackintosh et al. 1990, Hooser et al. 1991). Moreover, at sub-lethal doses, these toxins might act as tumor promoters (Ueno et al. 1996, Ding et al. 1999, Zhou et al. 2002).

Cylindrospermopsin (CYN) is a tricyclic alkaloid produced by Cylindrospermopsis (Ohtani et al. 1992, Berry and Lind 2010), Aphanizomenon (Banker et al. 1997, Preußel et al. 2006), Umezakia (Harada et al. 1994), Anabaena (Schembri et al. 2001, Spoof et al. 2006) and Lyngbya (Seifert et al. 2007). These are cytotoxins that irreversibly block protein synthesis, where the primary clinical symptoms are both hepatic and renal failure. They also act on tissues of the intestinal tract, vascular system and muscles (Terao et al. 1994, Falconer et al. 1999, Seawright et al. 1999, Froscio et al. 2008). Additionally, there are indications that CYN produces genotoxic, carcinogenic and mutagenic effects (Falconer and Humpage 2001, Saker et al. 2003). Following the first case of human intoxication provoked by CYN in Australia, 1979 (Hawkins et al. 1985), this toxin has been systematically found in other regions of the world (Carmichael et al. 2001, Li et al. 2001, Stirling and Quilliam 2001, Burns et al. 2002, Chonudomkul et al. 2004, Berry and Lind 2010), including Brazil (Bittencourt-Oliveira et al. 2011a).

Blooms of toxic cyanobacteria have been recorded worldwide and have become more numerous in both fresh and marine water (Hudnell et al. 2008, Paerl and Huisman 2009, Paerl et al. 2011, O’Neil et al. 2012). Parallel to an increase in documentation regarding these events, new species have been found to be responsible for these toxins (Bormans et al. 2005, Cox et al. 2005, Richardson et al. 2007, Berry and Lind 2010, Sant'Anna et al. 2011, Smith et al. 2011). As well, new occurrences of several toxins have been recorded in regions where they were historically thought not be present $\left(\mathrm{O}^{\prime}\right.$ Neil and Dennison 2005, Paul et al. 2005, Berry and Lind
2010, Bittencourt-Oliveira et al. 2011a, Smith et al. 2012, Mohamed and Al-Shehri 2013). With regard to Brazil, the observation of toxins from cyanobacteria in water intended for public drinking supply has been recorded in several regions, particularly in the south and the northeast (Molica et al. 2005, Anjos et al. 2006, Costa et al. 2006, Sotero-Santos et al. 2006, 2008, Moschini-Carlos et al. 2009, BittencourtOliveira et al. 2010, 2012a).

The monitoring relevance of cyanobacteria toxins in reservoirs for public supply is notorious. Brazil was the first country to enforce a specific legislation for the control of cyanobacteria and their toxins in water used for drinking supply (Brasil 2011). Monitoring of reservoirs requires cell counting of potentially toxic cyanobacteria and toxin quantification (New Zealand, Ministry of Health 2008, Brasil 2011, NHMRC, NRMMC 2011).

The most recurrent toxins in Brazilian water bodies are the MCs. However, the recent record of CYN in three reservoirs for public supply in the northeast region alerted to the possibility that it could be spreading (Bittencourt-Oliveira et al. 2011a).

Reservoirs in Pernambuco state were designed to fulfill different roles and multiple uses which, to some extent, make management difficult and, at the same time, increase the instability of the aquatic biota. These issues demand frequent environmental studies, plus investigation on the cyanobacteria communities and, in particular, their toxins. The occurrence of cyanobacteria toxins in these reservoirs (northeastern Brazil) was already recorded by a few authors using different techniques (Bouvy et al. 1999, Molica et al. 2005, BittencourtOliveira et al. 2010, 2011a, 2012a).

This study shows, for the first time, a wide and profound vision on the reservoirs in the northeastern region of Brazil with regard to cyanobacteria and their toxins. In addition to providing a glimpse of the current status for future research, this study can aid in comparative studies of diagnosis and monitoring, both on a 
regional and global scale, given the expansion of species of toxic cyanobacteria and their impact on the human population.

This study presents data gathered from 10 reservoirs of the state of Pernambuco, northeast of Brazil, destined for public drinking supply. The occurrence of MC and CYN, as well as the composition and quantification of the cyanobacteria community, is shown.

\section{MATERIALS AND METHODS}

STUDIED RESERVOIRS AND SAMPLINGS

The investigated reservoirs supply cities with high demographic densities. These reservoirs are located in regions where climates are warm but rain regimes can range from regular (Zona da Mata, Agreste) to sparse (Sertão, $37^{\circ} \mathrm{C}$ yearly average). Importance was given to reservoirs for public supply identified as those frequently having cyanobacteria blooms (Table I). The sampling sites were, preferentially, at the center of each reservoir.

Twenty three samples from 10 reservoirs in the state of Pernambuco (Table II) were collected, preferentially, in two different seasons, dry and rainy. Samples for cyanobacteria identification were gathered by surface dragging, using $20-\mu \mathrm{m}$ mesh plankton net or a wide neck bottle in the case of high population density. Samples for quantification analyses were gathered using a van Dorn bottle in the subsurface $(0.5 \mathrm{~m})$.

\section{TABLE I}

Reservoirs, coordinates, use, phytogeographic region, water capacity $\left(\mathrm{m}^{3}\right)$, maximum depth (m), target community and trophic state. S. Public Supply. W. Watering. F. Fishing. ND. No data.

\begin{tabular}{|c|c|c|c|c|c|c|}
\hline Reservoir (Use) & Coordinates & $\begin{array}{c}\text { Phytogeographic } \\
\text { Region }\end{array}$ & $\begin{array}{c}\text { Water } \\
\text { Capacity }\end{array}$ & $\begin{array}{l}\text { Max. } \\
\text { Depth }\end{array}$ & $\begin{array}{l}\text { Target } \\
\text { community }\end{array}$ & Trophic state \\
\hline Alagoinha (S, W) & $8^{\circ} 27^{\prime} 31.9^{\prime \prime} \mathrm{S}, 6^{\circ} 46^{\prime} 33.5^{\prime \prime} \mathrm{W}$ & Agreste & $2.0 \times 10^{4}$ & 5.0 & 11,886 & ND \\
\hline Arcoverde $(\mathrm{S}, \mathrm{W}, \mathrm{F})$ & $8^{\circ} 33^{\prime} 32.5^{\prime} \mathrm{S}, 6^{\circ} 59^{\prime} 07.5^{\prime \prime} \mathrm{W}$ & Agreste & $1.7 \times 10^{8}$ & 20.0 & 55,902 & Eutrophic $^{\mathrm{a}}$ \\
\hline Carpina (S, F) & $7^{\circ} 53^{\prime} 03.8^{\prime \prime} \mathrm{S}, 5^{\circ} 20^{\prime} 37.8^{\prime \prime} \mathrm{W}$ & Zona da Mata & $2.7 \times 10^{8}$ & 15.0 & 124,520 & Eutrophic $^{\mathrm{b}}$ \\
\hline Duas Unas $(\mathrm{S}, \mathrm{W}, \mathrm{F})$ & $8^{\circ} 05^{\prime} 02^{\prime \prime} \mathrm{S}, 35^{\circ} 30.6^{\prime \prime} \mathrm{W}$ & Zona da Mata & $2.4 \times 10^{7}$ & 14.0 & 914,144 & Eutrophic $^{c}$ \\
\hline Ingazeira $(\mathrm{S}, \mathrm{W}, \mathrm{F})$ & $8^{\circ} 36^{\prime} 41.2^{\prime \prime} \mathrm{S}, 6^{\circ} 54^{\prime} 23.7^{\prime \prime} \mathrm{W}$ & Agreste & $4.8 \times 10^{6}$ & 5.0 & 9,311 & Hypertrophic $^{\mathrm{d}^{*}}$ \\
\hline Ipojuca $(\mathrm{S}, \mathrm{F})$ & $8^{\circ} 20^{\prime} 43,7^{\prime \prime} \mathrm{S}, 36^{\circ} 22^{\prime} 31,5^{\prime \prime} \mathrm{W}$ & Agreste & $3.1 \times 10^{7}$ & 15.0 & 110,558 & Eutrophice \\
\hline Jucazinho (S, W, F) & $7^{\circ} 59^{\prime} 03^{\prime \prime} \mathrm{S}, 35^{\circ} 48^{\prime} 36.7^{\prime \prime} \mathrm{W}$ & Agreste & $3.2 \times 10^{8}$ & 40.0 & 55,766 & Eutrophic $^{c}$ \\
\hline Mundaú (S, W, F) & $8^{\circ} 56^{\prime} 42.8^{\prime \prime} \mathrm{S}, 36^{\circ} 29^{\prime} 27.4^{\prime \prime} \mathrm{W}$ & Agreste & $1.9 \times 10^{6}$ & 9.0 & 115,950 & Eutrophic $^{\mathrm{f}}$ \\
\hline Tapacurá $(\mathrm{S}, \mathrm{W}, \mathrm{F})$ & $8^{\circ} 02^{\prime} 31.9^{\prime \prime} \mathrm{S}, 35^{\circ} 11^{\prime} 46.5^{\prime \prime} \mathrm{W}$ & Zona da Mata & $9.4 \times 10^{7}$ & 9.7 & 940,224 & Eutrophic $^{c}$ \\
\hline Venturosa $(\mathrm{S}, \mathrm{W})$ & $8^{\circ} 34^{\prime} 43.6^{\prime \prime} \mathrm{S}, 36^{\circ} 52^{\prime} 47.3^{\prime \prime} \mathrm{W}$ & Agreste & $12.0 \times 10^{4}$ & 6.0 & 9,311 & Eutrophic $^{\mathrm{d}^{*}}$ \\
\hline
\end{tabular}

a. Bittencourt-Oliveira et al. (2012b); b. Moura et al. (2011); c. Dantas et al. (2012); d*. Trophic State Index calculated from data of chlorophyll $a$ of Bouvy et al. (2000) using trophic delineation according Forsberg and Ryding (1980); e. A.N. Moura (unpublished data); f. Dantas et al. (2010). 
TABLE II

Diversity, partial density $\left(\times 10^{6}\right.$ cell. $\left.\mathrm{mL}^{-1}\right)$, total density $\left(10^{6}\right.$ cell.mL $\left.\mathrm{m}^{-1}\right)$, partial biomass $\left(\mathrm{mg} . \mathrm{L}^{-1}\right)$ and total biomass of cyanobacteria in samples investigated. $(\%)$. Corresponding percentages of cell.mL $\mathrm{m}^{-1} \mathrm{or}$ mg. $\mathrm{L}^{-1}$ of cyanobacteria. (n). average number of cells per organism. Concentrations of microcystins (MC) and cylindrospermopsin (CYN)

(ng. $\mathrm{g}^{-1}$ freeze-dried cells) in the environmental samples obtained by ELISA (three replicates). SD. Standard deviations. $(*)$. Abundant species $\left({ }^{\circ}\right)$. Dominant species. (s). straight, (c). coiled. (-) absent.

\begin{tabular}{|c|c|c|c|c|c|c|c|c|c|}
\hline $\begin{array}{c}\text { Reservoir } \\
\text { (Date sampling) } \\
\text { Season }\end{array}$ & Cyanobacteria (n) & $\begin{array}{l}\text { Partial } \\
\text { Density }\end{array}$ & $\%$ & $\begin{array}{c}\text { Total } \\
\text { Density }\end{array}$ & $\begin{array}{c}\text { Partial } \\
\text { Biomass }\end{array}$ & $\%$ & $\begin{array}{c}\text { Total } \\
\text { Biomass }\end{array}$ & $\begin{array}{l}\text { MC } \\
\text { (SD) }\end{array}$ & $\begin{array}{l}\text { CYN } \\
\text { (SD) }\end{array}$ \\
\hline \multirow{6}{*}{$\begin{array}{c}\text { Alagoinha } \\
\text { (04.14.2009) } \\
\text { Rainy }\end{array}$} & $\begin{array}{l}\text { Cylindrospermopsis raciborskii } \\
\text { s, c (34)* }\end{array}$ & 2.16 & 42.06 & & 27.51 & 51.84 & \multirow{6}{*}{53.07} & \multirow{6}{*}{$\begin{array}{c}836,280.0 \\
( \pm 16,122.0)\end{array}$} & \multirow{6}{*}{-} \\
\hline & Microcystis aeruginosa $(420) *$ & 0.88 & 17.18 & & 8.36 & 15.75 & & & \\
\hline & Microcystis panniformis (419)* & 1.21 & 23.49 & 5.14 & 6.55 & 12.34 & & & \\
\hline & Gleitlerinema amphibium (61) & 0.51 & 9.95 & & 5.11 & 9.63 & & & \\
\hline & Planktothrix agardhii (57) & 0.28 & 5.52 & & 4.43 & 8.35 & & & \\
\hline & Others & 0.093 & 1.80 & & 1.11 & 2.09 & & & \\
\hline \multirow{4}{*}{$\begin{array}{c}\text { Alagoinha } \\
(10.13 .2009) \\
\text { Dry }\end{array}$} & Planktothrix agardhii $(75)^{\circ}$ & 10.43 & 89.67 & & 163.10 & 86.88 & \multirow{4}{*}{187.73} & & \multirow{4}{*}{-} \\
\hline & $\begin{array}{l}\text { Sphaerospermopsis } \\
\text { aphanizomenoides }(45)\end{array}$ & 0.27 & 2.28 & 11.63 & 15.25 & 8.12 & & $13,542.0$ & \\
\hline & $\begin{array}{l}\text { Cylindrospermopsis raciborskii } \\
\mathbf{s}(30)\end{array}$ & 0.41 & 3.54 & & 5.25 & 2.80 & & $( \pm 118.8)$ & \\
\hline & Others & 0.52 & 4.51 & & 4.13 & 2.20 & & & \\
\hline \multirow{3}{*}{$\begin{array}{c}\text { Arcoverde } \\
(05.12 .2009) \\
\text { Rainy }\end{array}$} & $\begin{array}{l}\text { Cylindrospermopis raciborskii } \\
\mathbf{s , c}(26)^{\circ}\end{array}$ & 95.70 & 77.93 & & 12.44 & 82.98 & \multirow{3}{*}{14.99} & \multirow[t]{3}{*}{306.1} & \multirow{3}{*}{$( \pm 4.0)$} \\
\hline & Geitlerinema amphibium (40) & 24.20 & 19.71 & 122.80 & 2.42 & 16.14 & & & \\
\hline & Others & 2.90 & 2.36 & & 0.13 & 0.88 & & & \\
\hline \multirow{5}{*}{$\begin{array}{c}\text { Carpina } \\
(04.06 .2009) \\
\text { Rainy }\end{array}$} & Planktothrix agardhii $(81)^{\circ}$ & 5.96 & 74.63 & & 77.50 & 69.44 & \multirow{5}{*}{111.61} & \multirow{5}{*}{135.7} & \multirow{5}{*}{-} \\
\hline & $\begin{array}{l}\text { Cylindrospermopsis raciborskii } \\
\mathbf{s ,} \mathbf{c}(24)\end{array}$ & 1.10 & 13.74 & 7.99 & 8.78 & 7.87 & & & \\
\hline & $\begin{array}{l}\text { Sphaerospermopsis } \\
\text { aphanizomenoides (44) }\end{array}$ & 0.19 & 2.33 & & 8.77 & 7.86 & & & \\
\hline & Geitlerinema amphibium (85) & 0.32 & 4.05 & & 4.21 & 3.77 & & & \\
\hline & Others & 0.42 & 5.25 & & 12.36 & 11.07 & & & \\
\hline \multirow{4}{*}{$\begin{array}{c}\text { Carpina } \\
(\mathbf{1 0 . 0 6 . 2 0 0 9 )} \\
\text { Dry }\end{array}$} & Planktothrix agardhii $(80)^{\circ}$ & 12.90 & 92.18 & & 167.51 & 88.59 & \multirow{4}{*}{189.08} & & \multirow{4}{*}{-} \\
\hline & Planktothrix isothrix (88) & 0.43 & 3.04 & 14.0 & 7.65 & 4.05 & & 93.9 & \\
\hline & Geitlerinema amphibium (174) & 0.42 & 3.01 & & 5.46 & 2.89 & & $( \pm 8.3)$ & \\
\hline & Others & 0.25 & 1.77 & & 8.45 & 4.47 & & & \\
\hline \multirow{3}{*}{$\begin{array}{c}\text { Duas Unas } \\
(03.10 .2009) \\
\text { Dry }\end{array}$} & $\begin{array}{l}\text { Sphaerospermopsis } \\
\text { aphanizomenoides }(53)^{\circ}\end{array}$ & 2.10 & 92.84 & & 84.53 & 96.78 & \multirow{3}{*}{87.34} & & \multirow{3}{*}{-} \\
\hline & $\begin{array}{l}\text { Cylindrospermopsis raciborskii } \\
\text { s (24) }\end{array}$ & 0.09 & 3.92 & 2.27 & 1.07 & 1.23 & & 62.7 & \\
\hline & Others & 0.07 & 3.24 & & 1.74 & 1.99 & & $( \pm 4.4)$ & \\
\hline \multirow{2}{*}{$\begin{array}{c}\text { Duas Unas } \\
(05.04 .2009) \\
\text { Rainy }\end{array}$} & Microcystis panniformis $(540)^{\circ}$ & $3,356.7$ & 99.15 & $3,385.50$ & 31.79 & 98.92 & \multirow[t]{2}{*}{32.13} & 170.3 & 143.0 \\
\hline & $\begin{array}{l}\text { Cylindrospermopsis raciborskii } \\
\mathbf{s}(30)\end{array}$ & 28.8 & 0.85 & & 0.34 & 1.07 & & $( \pm 10.0)$ & $( \pm 14.0)$ \\
\hline
\end{tabular}


TABLE II (continuation)

\begin{tabular}{|c|c|c|c|c|c|c|c|c|c|}
\hline $\begin{array}{c}\text { Reservoir } \\
\text { (Date sampling) } \\
\text { Season }\end{array}$ & Cyanobacteria $(n)$ & $\begin{array}{l}\text { Partial } \\
\text { Density }\end{array}$ & $\%$ & $\begin{array}{c}\text { Total } \\
\text { Density }\end{array}$ & $\begin{array}{c}\text { Partial } \\
\text { Biomass }\end{array}$ & $\%$ & $\begin{array}{c}\text { Total } \\
\text { Biomass }\end{array}$ & $\begin{array}{l}\text { MC } \\
\text { (SD) }\end{array}$ & $\begin{array}{l}\text { CYN } \\
\text { (SD) }\end{array}$ \\
\hline \multirow{4}{*}{$\begin{array}{c}\text { Ingazeira } \\
(04.14 .2009) \\
\text { Rainy }\end{array}$} & Planktothrix agardhii $(77)^{\circ}$ & 10.30 & 69.70 & & 160.58 & 76.77 & \multirow{4}{*}{209.18} & & \multirow{4}{*}{-} \\
\hline & $\begin{array}{l}\text { Cylindrospermopsis raciborskii } \\
\mathbf{s ,} \mathbf{c}(18) *\end{array}$ & 2.47 & 16.71 & 14.78 & 32.09 & 15.34 & & & \\
\hline & Geitlerinema amphibium (117) & 1.64 & 11.10 & & 12.32 & 5.89 & & & \\
\hline & Others & 0.37 & 2.48 & & 4.19 & 2.00 & & & \\
\hline \multirow{4}{*}{$\begin{array}{c}\text { Ingazeira } \\
(10.13 .2009) \\
\text { Dry }\end{array}$} & Geitlerinema amphibium (117) ${ }^{\circ}$ & 4.26 & 52.76 & & 52.43 & 39.59 & \multirow{4}{*}{132.44} & & \\
\hline & Planktothrix agardhii $(77) *$ & 2.64 & 32.67 & 8.07 & 39.53 & 29.85 & & $2,032.0$ & \\
\hline & $\begin{array}{l}\text { Cylindrospermopsis raciborskii } \\
\text { s, c (18) }\end{array}$ & 0.87 & 10.79 & & 27.93 & 21.09 & & $( \pm 78.4)$ & \\
\hline & Others & 0.30 & 3.78 & & 12.54 & 9.47 & & & \\
\hline \multirow{3}{*}{$\begin{array}{c}\text { Ipojuca } \\
(05.26 .2010) \\
\text { Dry }\end{array}$} & Planktothrix isothrix $(115)^{\circ}$ & 1.33 & 92.37 & & 25.41 & 95.81 & \multirow{3}{*}{26.52} & & \multirow{3}{*}{$\begin{array}{c}3.43 \\
( \pm 0.5)\end{array}$} \\
\hline & Geitlerinema amphibium (54) & 0.055 & 3.77 & 1.44 & 0.49 & 1.85 & & - & \\
\hline & Others & 0.053 & 3.86 & & 0.62 & 2.34 & & & \\
\hline \multirow{3}{*}{$\begin{array}{c}\text { Ipojuca } \\
(05.03 .2010) \\
\text { Rainy }\end{array}$} & Planktothrix isothrix $(115)^{\circ}$ & 1.40 & 91.69 & & 26.58 & 93.86 & \multirow{3}{*}{28.32} & & \multirow{3}{*}{201.5} \\
\hline & $\begin{array}{l}\text { Cylindrospermopsis raciborskii } \\
\text { s, c (35) }\end{array}$ & 0.12 & 7.63 & 1.53 & 1.16 & 4.10 & & 10.3 & \\
\hline & Others & 0.01 & 0.68 & & 0.58 & 2.05 & & $( \pm 1.1)$ & \\
\hline \multirow{4}{*}{$\begin{array}{c}\text { Jucazinho } \\
\text { (02.17.2009) } \\
\text { Rainy }\end{array}$} & Planktothrix agardhii $(118)^{\circ}$ & 22.20 & 41.57 & & 31.08 & 30.73 & \multirow{4}{*}{101.13} & & \multirow{4}{*}{76.3} \\
\hline & $\begin{array}{l}\text { Sphaerospermopsis } \\
\text { aphanizomenoides (41) }\end{array}$ & 19.5 & 36.52 & & 58.93 & 58.27 & & 64.0 & \\
\hline & $\begin{array}{l}\text { Cylindrospermopsis raciborskii } \\
\text { s, c (16) }\end{array}$ & 8.4 & 15.73 & & 8.40 & 8.31 & & $( \pm 8.8)$ & \\
\hline & Others & 3.3 & 6.18 & 53.40 & 2.72 & 2.69 & & & \\
\hline \multirow{5}{*}{$\begin{array}{c}\text { Jucazinho } \\
\left(\begin{array}{c}03.24 .2009) \\
\text { Dry }\end{array}\right.\end{array}$} & Planktothrix agardhii (117) ${ }^{\circ}$ & 101.6 & 52.13 & & 14.22 & 47.46 & \multirow{5}{*}{29.97} & & \multirow{5}{*}{$( \pm 279.0)$} \\
\hline & Geitlerinema amphibium (43)* & 41.2 & 21.14 & 194.90 & 4.94 & 16.50 & & & \\
\hline & $\begin{array}{l}\text { Sphaerospermopsis } \\
\text { aphanizomenoides (41) }\end{array}$ & 28.5 & 14.62 & & 8.61 & 28.74 & & 489.2 & \\
\hline & $\begin{array}{l}\text { Cylindrospermopsis raciborskii } \\
\text { s, c (16) }\end{array}$ & 15.2 & 7.80 & & 1.52 & 5.07 & & $( \pm 44.1)$ & \\
\hline & Others & 8.4 & 4.31 & & 0.67 & 2.24 & & & \\
\hline \multirow{4}{*}{$\begin{array}{c}\text { Jucazinho } \\
(\mathbf{1 0 . 2 7 . 2 0 0 9 )} \\
\text { Dry }\end{array}$} & Planktothrix agardhii (118) ${ }^{\circ}$ & 61.90 & 79.77 & & 86.66 & 80.13 & \multirow{4}{*}{108.15} & & \multirow{4}{*}{0.5} \\
\hline & Geitlerinema amphibium (43) & 6.90 & 8.89 & 77.60 & 8.28 & 7.66 & & 117.7 & \\
\hline & $\begin{array}{l}\text { Cylindrospermopsis raciborskii } \\
\text { s, c (16) }\end{array}$ & 6.40 & 8.25 & & 6.40 & 5.92 & & $( \pm 8.7)$ & \\
\hline & Others & 2.40 & 3.09 & & 6.81 & 6.30 & & & \\
\hline \multirow{5}{*}{$\begin{array}{c}\text { Jucazinho } \\
(04.28 .2009) \\
\text { Rainy }\end{array}$} & Planktothrix agardhii (117) ${ }^{\circ}$ & 333.4 & 74.42 & & 46.68 & 71.53 & \multirow{5}{*}{65.25} & & \multirow{5}{*}{$\begin{array}{c}201.4 \\
( \pm 90.0)\end{array}$} \\
\hline & Geitlerinema amphibium (43) & 63.7 & 14.22 & 448.00 & 7.64 & 11.71 & & 83.6 & \\
\hline & $\begin{array}{l}\text { Sphaerospermopsis } \\
\text { aphanizomenoides (41) }\end{array}$ & 29.3 & 6.54 & & 8.85 & 13.57 & & $( \pm 3.7)$ & \\
\hline & $\begin{array}{l}\text { Cylindrospermopsis raciborskii } \\
\text { s, c (16) }\end{array}$ & 17.4 & 3.88 & & 1.74 & 2.67 & & & \\
\hline & Others & 4.20 & 0.94 & & 0.34 & 0.51 & & & \\
\hline
\end{tabular}


TABLE II (continuation)

\begin{tabular}{|c|c|c|c|c|c|c|c|c|c|}
\hline $\begin{array}{c}\text { Reservoir } \\
\text { (Date sampling) } \\
\text { Season }\end{array}$ & Cyanobacteria (n) & $\begin{array}{l}\text { Partial } \\
\text { Density }\end{array}$ & $\%$ & $\begin{array}{c}\text { Total } \\
\text { Density }\end{array}$ & $\begin{array}{c}\text { Partial } \\
\text { Biomass }\end{array}$ & $\%$ & $\begin{array}{c}\text { Total } \\
\text { Biomass }\end{array}$ & $\begin{array}{l}\text { MC } \\
\text { (SD) }\end{array}$ & $\begin{array}{l}\text { CYN } \\
\text { (SD) }\end{array}$ \\
\hline \multirow{2}{*}{$\begin{array}{c}\text { Mundaú } \\
\text { (09.02.2008) } \\
\text { Rainy }\end{array}$} & Microcystis panniformis $(827)^{\circ}$ & 1.43 & 99.66 & & 7.69 & 95.17 & & 630.9 & \\
\hline & Others & 0.05 & 3.34 & 1.48 & 0.39 & 4.83 & 8.08 & $( \pm 41.1)$ & - \\
\hline \multirow{3}{*}{$\begin{array}{c}\text { Mundaú } \\
\text { (03.17.2009) } \\
\text { Dry }\end{array}$} & Microcystis panniformis $(827)^{\circ}$ & 16.11 & 87.92 & & 86.68 & 77.13 & & & \\
\hline & Others & 0.63 & 3.42 & 18.3 & 13.02 & 11.59 & 112.38 & $16,858.4$ & - \\
\hline & $\begin{array}{l}\text { Cylindrospermopsis raciborskii } \\
\text { s, c (17) }\end{array}$ & 1.59 & 8.66 & & 12.69 & 11.29 & & $( \pm 1,314.1)$ & \\
\hline \multirow{3}{*}{$\begin{array}{c}\text { Mundaú } \\
\text { (05.05.2009) } \\
\text { Rainy }\end{array}$} & Microcystis panniformis $(1143)^{\circ}$ & 7.21 & 75.99 & & 38.79 & 68.73 & & $3,316.5$ & \\
\hline & $\begin{array}{l}\text { Cylindrospermopsis raciborskii } \\
\text { s, c (23) }\end{array}$ & 2.08 & 21.97 & 9.49 & 16.67 & 29.54 & 56.42 & $( \pm 271.5)$ & - \\
\hline & Others & 0.21 & 2.05 & & 0.96 & 1.70 & & & \\
\hline \multirow{3}{*}{$\begin{array}{c}\text { Mundaú } \\
(11.09 .2009) \\
\text { Dry }\end{array}$} & Microcystis panniformis $(1143)^{\circ}$ & 8.27 & 85.88 & & 44.48 & 81.38 & & & \\
\hline & $\begin{array}{l}\text { Cylindrospermopsis raciborskii } \\
\mathbf{s , c}(17)\end{array}$ & 1.16 & 12.05 & 9.63 & 9.28 & 16.98 & 54.66 & $2,366.1$ & - \\
\hline & Others & 0.20 & 2.07 & & 0.90 & 1.65 & & $( \pm 114.5)$ & \\
\hline \multirow{3}{*}{$\begin{array}{c}\text { Tapacurá } \\
(03.10 .2009) \\
\text { Dry }\end{array}$} & Microcystis panniformis (78) ${ }^{\circ}$ & 1.49 & 71.95 & & 39.33 & 85.93 & & 52.7 & \\
\hline & Planktothrix agardhii (73) & 0.26 & 12.59 & 2.07 & 3.65 & 7.97 & 45.77 & $( \pm 5.4)$ & - \\
\hline & Others & 0.32 & 15.48 & & 2.79 & 6.1 & & & \\
\hline \multirow{3}{*}{$\begin{array}{c}\text { Tapacurá } \\
(05.04 .2009) \\
\text { Rainy }\end{array}$} & Microcystis panniformis $(8653)^{\circ}$ & 2.27 & 88.91 & & 59.80 & 93.54 & & 30.4 & \\
\hline & Planktothrix agardhii (73) & 0.13 & 5.11 & 2.55 & 1.82 & 2.85 & 63.93 & $( \pm 1.5)$ & - \\
\hline & Others & 0.15 & 5.98 & & 2.31 & 3.61 & & & \\
\hline \multirow{4}{*}{$\begin{array}{c}\text { Tapacurá } \\
(\mathbf{1 0 . 0 5 . 2 0 0 9 )} \\
\text { Dry }\end{array}$} & 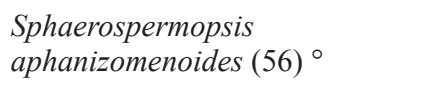 & 1.73 & 43.10 & & 70.85 & 65.64 & & & \\
\hline & Microcystis panniformis (3593)* & 0.63 & 15.60 & 4.02 & 16.54 & 15.33 & 107.93 & 62.3 & - \\
\hline & $\begin{array}{l}\text { Cylindrospermopsis raciborskii } \\
\mathbf{s ,} \mathbf{c}(16)\end{array}$ & 0.63 & 15.64 & & 9.43 & 8.74 & & $( \pm 1.1)$ & \\
\hline & Others & 1.03 & 25.66 & & 11.11 & 10.3 & & & \\
\hline \multirow{2}{*}{$\begin{array}{c}\text { Venturosa } \\
(10.13 .2009) \\
\text { Dry }\end{array}$} & Merismopedia tenuissima $(16)^{\circ}$ & 11.76 & 99.95 & 11.77 & 19.05 & 99.57 & 19.13 & 17.3 & - \\
\hline & Others & 0.006 & 0.05 & & 0.08 & 0.43 & & $( \pm 1.4)$ & \\
\hline
\end{tabular}

IDENTIFICATION AND EVALUATION OF DENSITY AND BIOMASS

Cyanobacteria identification was carried out under a light microscope (Nikon YS100, Melville, NY, USA), and by comparison with specialized literature. Samples for density evaluation (cells. $\mathrm{mL}^{-1}$ ) were maintained in acetic Lugol's solution and settled in counting chamber according to Utermöhl (1958). Densities were converted into biovolume following the procedure in Hillebrand et al. (1999) and transformed into biomass, assuming a mass density of $1 \mathrm{mg} . \mathrm{L}^{-1}$ (Wetzel and Likens 2000). Average values were used for biovolume measurement ( $n=50$, for abundant species and $n=10$, for less frequent species). Abundance and dominance were evaluated according to Lobo and Leighton (1986). Abundant species were those with densities above the community average density, while dominant species were those with density surpassing $50 \%$ of the community total density. 
MICROCYSTIN AND CYLINDROSPERMOPSIN ANALYSIS BY IMMUNOASSAY METHOD

Samples with approximately $20 \mathrm{~L}$ were collected, centrifuged, frozen in liquid nitrogen, and lyophilized. The lyophilized cells from environmental samples ( $\sim 10 \mathrm{mg}$ ) with $3 \mathrm{~mL}$ of Milli-Q water were disrupted by ultrasonication (Microson Ultrasonic Cell Disruptor, Misonix, USA) and used directly for analysis of MC and CYN. Toxin quantification was carried out using a commercial ELISA kit (Beacon Analytical Systems Inc., Portland, ME, USA), following the manufacturer's protocols. The high and low detection limits for MC and CYN by ELISA were 2.0 and $0.1 \mu \mathrm{g} . \mathrm{L}^{-1}$, respectively. Negative and positive controls for the ELISA analysis are included in the commercial kit. Triplicate analyses were performed and the values were averaged.

\section{RESULTS}

Occurrence of MC was found in all reservoirs (Table II). Only in one sample, out of 23 samples MC was absent (Ipojuca reservoir, 5/26/2010). It was found that in all samples cyanobacteria reported in literature as potentially toxin-producing (MC, CYN and SX), except for M. tenuissima. With respect to CYN, eight out of the 23 analyzed samples were positive in four reservoirs (Arcoverde, Duas Unas, Ipojuca, and Jucazinho). In these reservoirs MC and CYN were found simultaneously.

Diversity of cyanobacteria communities, their dominant and abundant taxa, densities and biomasses (total and partial), as well as the presence of MC and CYN in 23 samples were in the Table II. Cyanobacteria communities in the reservoirs were constituted, basically, by Cylindrospermopsis raciborskii (Woloszynka) Seenayya \& Subba Raju, Planktothrix agardhii (Gomont) Anagnostidis \& Komárek, Sphaerospermopsis aphanizomenoides (Forti) Zapomělová, Jezberová, Hrouzek, Hizem, Reháková \& Komárková and Geitlerinema amphibium (Gomont) Anagnostidis. C. raciborskii and $P$. agardhii were dominant and abundant in five reservoirs, which had MC or CYN. Planktothrix species (P. agardhii and P. isothrix (Skuja) Komárek $\&$ Komárková) were dominant in five reservoirs and were responsible for more than $50 \%$ of cyanobacteria total biomass. In addition, they were the highest occurring genus in blooms, followed by Microcystis panniformis Komárek et al. in four reservoirs. The species Planktothrix agardhii and Microcystis panniformis reached the highest densities, surpassing $10^{7}$ cells.mL ${ }^{-1}$. Planktothrix agardhii, $P$. isothrix, Sphaerospermopsis aphanizomenoides, M. panniformis and Merismopedia tenuissima Lemmermann constituted monospecific blooms, accounted for up to $85 \%$ of cyanobacteria total biomass in the sample (Table II).

Generally speaking, there was no correlation between dry and rainy seasons, increase or decrease of superficial cyanobacteria biomasses or densities, in the reservoirs.

\section{DISCUSSION}

Cyanotoxins were found in $100 \%$ of the set of reservoirs (ten), where MCs were detected in $95.65 \%$ of the samples investigated for MC and CYN.

Early reports showed that toxic cyanobacteria have dominated in warmer waters of the tropics and subtropics. Within the regions with tropical and sub-tropical climates, Australia shows some similarity to the northeast of Brazil, due to its area, its many reservoirs, shallows, rivers and, mainly, its historical records of frequent blooms of toxic cyanobacteria (Burford et al. 2007, Burford and Davis 2011). Toxin production records are common in Brazil and Australia, and is less in other areas of the world (Fastner et al. 2003, Saker et al. 2003, Burford and Davis 2011).

Brazil has a history of contamination of freshwater systems by cyanotoxins (Teixeira et al. 1993, Jochimsen et al. 1998). Over the last ten years, there have been frequent records of cyanobacteria blooms in reservoirs throughout the country (Anjos 
et al. 2006, Costa et al. 2006, Chellappa et al. 2008, Dantas et al. 2011, Bittencourt-Oliveira et al. 2011a, b, 2012a, Piccin-Santos and BittencourtOliveira 2012).

In spite of monospecific bloom occurrences in the reservoirs of Ingazeira, Jucazinho, Mundaú and Tapacurá, those blooms constituted by more than one species were the most frequent. Costa et al. (2006), Bittencourt-Oliveira et al. (2011a, 2012b), Moura et al. (2011), and Lira et al. (2011) reported multi-species blooms of cyanobacteria constituted mostly by the genera Planktothrix and Cylindrospermopsis, with possible alternation of predominance according to the climate conditions.

Apart from high temperatures, blooms in the northeast of Brazil are also enhanced by the hydric regime characterized by long and accentuated dry periods and by the length of time that the water remains in the reservoirs. Conditions associated with dry and rainy seasons, such as stratification and de-stratification of water bodies, as well as water turbidity, are appointed as favoring factors, allowing the establishment of other species with high population densities and forming multispecies blooms of cyanobacteria (Dantas et al. 2011, Bittencourt-Oliveira et al. 2012b). Bittencourt-Oliveira et al. (2012b) observed in the Arcoverde reservoir an overwhelming increase of $C$. raciborskii populations during the dry season (destratification), characterized by thermally stratified water where, in the rainy season, the cyanobacteria biomass dropped substantially due to the presence of other cyanobacteria in the community. These authors pointed out that species being favored by stratification cannot be considered a rule because in other reservoirs in the same region the population density of $C$. raciborskii increased all through the thermal de-stratification. These differences in the behavior of populations of cyanobacteria, particularly of $C$. raciborskii, also vary between Brazilian and Australian water bodies (Hawkins and Grifftiths 1993, Bittencourt-Oliveira et al. 2011b).
In all samples with MCs, potentially toxic cyanobacteria were found, as described in the literature. The single exception of nontoxic cyanobacterium was $M$. tenuissima in the Venturosa reservoir (10.13.2009), even in high densities. Despite the high cellular density, the biomass was low (19.05 mg.L $\left.{ }^{-1}\right)$. Picoplanktonic cyanobacteria, such as Aphanocapsa cumulus Komárek \& Cronberg (Domingos et al. 1999) and Epigloeosphaera brasilica Azevedo et al. (Bittencourt-Oliveira et al. 2012a), were already reported in Brazil as toxin-producing. It should be emphasized that no cyanobacteria have to be discarded with respect to toxin production.

Diversity of the cyanobacteria community, mainly constituted by toxin-producing species (Cylindrospermopsis raciborskii, Planktohrix agardhii, Sphaerospermopsis aphanizomenoides and Geitlerinema amphibium), was similar in all reservoirs, except for Merismopedia tenuissima (Venturosa reservoir). Such homogeneity of cyanobacteria communities in reservoirs of the state of Pernambuco corroborate previous studies (Bouvy et al. 2000, 2003, Dantas et al. 2011, Lira et al. 2011, Moura et al. 2011, Bittencourt-Oliveira et al. 2011a, b, 2012a).

In the studied Brazilian reservoirs, $C$. raciborskii shared dominance with species of Planktothrix, whereas P. agardhii and P. isothrix formed monospecific blooms which accounted for up to $85 \%$ of the total cyanobacteria biomass in the sample (Table II). Cylindrospermopsis raciborskii, Sphaerospermopsis aphanizomenoides previously enominated Aphanizomenon aphanizomenoides (Zapomělová et al. 2009, 2010), G. amphibium, Microcystis and Planktothrix species are potentially microcystin, cylindrospermopsin and saxitoxinproducing. On the other hand, of the mentioned species, only C. raciborskii (Ohtani et al. 1992, Li et al. 2001) and species of the genus Sphaerospermopsis (Aphanizomenon spp.) (Preußel et al. 2006, Yilmaz et al. 2008) have been described as producers of CYN.

Although populations of $C$. raciborskii and Aphanizomenon/Sphaerospermopsis spp. are 
common in the country, the toxin CYN was found only recently in Brazil (Bittencourt-Oliveira et al. 2011a). However, even though CYN occurs in reservoirs with high densities of C. raciborskii and Sphaerospermopsis no CYN-producing strain has been isolated in order to verify which species was responsible for producing this toxin. In Brazil no strain was reported as CYN-producing.

The Jucazinho reservoir had the greatest concentrations of CYN, associated with a high biomass of Planktothrix agardhii, Sphaerospermopsis aphanizomenoides and Geitlerinema amphibium. P. agardhii was the predominant species in three out of four samples in this reservoir, with total cyanobacteria biomass ranging from 30.73 to $80.13 \%$, but there were no reports of an association with CYN production.

The sample from the Arcoverde reservoir taken on May 12, 2009 was the only one with an expressive predominance of $C$. raciborskii and also had the lowest concentrations of CYN (33.3 ng. $\mathrm{g}^{-1}$ freezedried cells). Therefore, biomass and density values of MC and CYN-producing cyanobacteria were not proportional to the concentrations of the measured toxins. This fact was already reported in previous studies undertaken in the reservoirs of the region (Piccin-Santos and Bittencourt-Oliveira 2012).

\section{CONCLUSION}

The similarity of cyanobacteria communities in the investigated reservoirs, associated with both the occurrence of $\mathrm{MC}$ and $\mathrm{CYN}$ and adequate environmental conditions, favors geographic spreading of toxic blooms in the region. This circumstance emphasizes the need for increased monitoring of MC and CYN in drinking supply reservoirs in Brazil.

\section{ACKNOWLEDGMENTS}

This research was supported by grants from Conselho Nacional de Desenvolvimento Científico e Tecnológico (CNPq Proc. 576890/2008-1 and 301739/2011-0).

\section{RESUMO}

O Brasil tem um histórico de florações e contaminações por toxinas de cianobactérias nos seus ecossistemas de água doce. A relevância do monitoramento de toxinas de cianobactérias em reservatórios de abastecimento público é notória, devido à sua alta toxicidade para mamíferos, inclusive seres humanos. As cianotoxinas mais recorrentes em corpos de água brasileiros são microcistinas (MC). No entanto, o registro recente de cilindrospermopsina (CYN) no nordeste, estado de Pernambuco, nos alerta para a possibilidade do aumento de novas ocorrências. Este estudo relata a ocorrência de MC e CYN, quantificadas por ELISA, em 10 reservatórios destinados ao abastecimento público do nordeste do Brasil. Também são apresentadas a composição e a quantificação da comunidade de cianobactérias associadas a estes corpos de água. Das 23 amostras investigadas, 22 e 8 foram positivas em relação, respectivamente, a $\mathrm{MC}$ e CYN. Considerando a similaridade das comunidades de cianobactérias encontradas nos reservatórios de Pernambuco e a presença de espécies potencialmente produtoras de MC e CYN, nós sugerimos que uma expansão geográfica possa ser favorecida por esses fatores. Estas questões enfatizam a necessidade de aumentar o monitoramento de MC e CYN em reservatórios de abastecimento público no Brasil.

Palavras-chave: floração, algas nocivas, monitoramento, toxinas, qualidade de água.

\section{REFERENCES}

Anjos FM, Bittencourt-Oliveira MC, Zajac MP, Hiller S, Christian B, ERler K, Luckas B AND Pinto E. 2006. Detection of harmful cyanobacteria and their toxins by both PCR amplification and LC-MS during a bloom event. Toxicon 48: 239-245.

Banker R, CARMEli S, Hadas O, Teltsch B, Porat R AND SUKENIK A. 1997. Identification of cylindrospermopsin in Aphanizomenon ovalisporum (Cyanophyceae) isolated from Lake Kinneret, Israel. J Phycol 33: 613-616.

BERRY JP AND LIND O. 2010. First evidence of "paralytic shellfish toxins" and cylindrospermopsin in a Mexican freshwater system, Lago Catemaco, and apparent bioaccumulation of the toxins in "tegogolo" snails (Pomacea patula catemacensis). Toxicon 55: 930-938. 
BITTENCOURT-OliveIra MC, DiAs SN, MOURA AN, CORDEIROARAÚJO MK AND DANTAS EW. 2012b. Seasonal dynamics of cyanobacteria in a eutrophic reservoir (Arcoverde) in the semi-arid region of Brazil. Braz J Biol 72: 533-544.

Bittencourt-Oliveira MC, Moura AN, Hereman TC AND DANTAS EW. 2011b. Increase in straight and coiled Cylindrospermopsis raciborskii (Cyanobacteria) populations under conditions of thermal de-stratification in a shallow tropical reservoir. J Water Resour Prot 3: 245-252.

BitTENCOURT-OliveIRA MC, PICCIN-SANTOS V AND GOUVÊABARROS S. 2012a. Microcystin-producing genotypes from cyanobacteria in Brazilian reservoirs. Environ Toxicol 27: 461-471.

Bittencourt-Oliveira MC, Piccin-SAntos V, KuJbida P AND MoURA AN. 2011a. Cylindrospermopsin in water supply reservoirs in Brazil determined by immunochemical and molecular methods. J Water Resour Prot 3: 349-355.

BitTencourt-Oliveira MC, SANTOS DMS AND Moura AN. 2010. Toxic cyanobacteria in reservoirs in Northeastern Brazil: detection using a molecular method. Braz J Biol 70: 1005-1010.

BORMANS M, FORD PW AND FABBRO L. 2005. Spatial and temporal variability in cyanobacteria populations controlled by physical processes. J Plankton Res 27: 61-70.

BOUVY MA, FALCÃO D, MARINHO M, PAGANO M AND MOURA AN. 2000. Occurrence of Cylindrospermopsis (Cyanobacteria) in 39 Brazilian tropical reservoirs during the 1998 drought. Aquat Microb Ecol 23: 13-27.

BOUVy MA, MOLICA R, OLIVEIRA S, MARINHO M AND BEKER B. 1999. Dynamics of a toxic Cyanobacterial bloom (Cylindrospermopsis raciborskii) in a shallow reservoir in the Semi-Arid region of Northeast Brazil. Aquat Microb Ecol 20: 285-297.

BOUVy MA, NASCIMENTO SM, MOLICA RJR AND FERREIRA A. 2003. Limnological features in Tapacurá reservoir (northeast Brazil) during a severe drought. Hydrobiologia 493: $115-130$.

BRASIL. 2011. Regulation MS No 2914, "Guidelines for drinking Water Quality”, Official Law Report's, 12, December 2011

BURFORD MA AND DAVIS TW. 2011. Physical and chemical processes promoting dominance of the toxic cyanobacterium Cylindrospermopsis raciborskii. Chinese J Oceanol Limnol 29: 883-891.

BURFORD MA, JOHNSON SA, COOK AJ, PACKER TV, TAYLOR BM AND TOWNSLEY ER. 2007. Correlations between watershed and reservoir characteristics, and algal blooms in subtropical reservoirs. Water Res 41: 4105-4114.

BURNS J, WILLIAMS C AND CHAPMAN A. 2002. Cyanobacteria and their toxins in Florida surface waters. In: JOHNSON D and HARBISON RD (Eds), Proceedings of Health Effects of Exposure to Cyanobacteria Toxins. Saratoga: State of Science 13/14: 16-21.

CARMichael WW. 1994. The Toxins of Cyanobacteria. Sci Am 270: 78-86.
CARMICHAEl WW, AZEVEDO SMFOO, AN JS, MOLICA RJR, JOCHIMSEN EM, LAU S, RINEHART KL, SHAW GR AND EAGLESHAM GK. 2001. Human fatalities from cyanobacteria, chemical and biological evidence for cyanotoxins. Environ Health Perspect 109: 663-668.

ChEllapPaNT, BorbA JMAND Rocha O. 2008. Phytoplankton community and physical-chemical characteristics of water in the public reservoir of Cruzeta, RN, Brazil. Braz J Biol 68: 477-494.

Chonudomkul D, Yongmanitchai W, Theeragool G, KAWACHI M, KASAI F, KAYA KAND WATANABE MM. 2004. Morphology, genetic diversity, temperature tolerance and toxicity of Cylindrospermopsis raciborskii (Nostocales, Cyanobacteria) strains from Thailand and Japan. FEMS Microbiol Ecol 48: 345-355.

Clark SP, DAVIS MA, RYAN TP, SEARFoss GH AND HoOSER SB. 2007. Hepatic gene expression changes in mice associated with prolonged sublethal microcystin exposure. Toxicol Pathol 35: 594-605.

Costa ias, Azevedo SMFo, Senna PAC, Bernardo RR, COSTA SM AND CHELlAPPA NT. 2006. Occurrence of toxin-producing cyanobacteria blooms in a Brazilian semiarid reservoir. Braz J Biol 66: 211-219.

COX PA, BANACK SA AND MURCH SJ. 2003. Biomagnification of cyanobacterial neurotoxins and neurodegenerative disease among the Chamorro people of Guam. Proc Natl Acad Sci USA 100: 13380-13383.

COX PA ET AL. 2005. Diverse taxa of cyanobacteria produceN-methylamino-L-alanine, a neurotoxic amino acid. Proc Natl Acad Sci USA 102: 5074-5078.

Dantas EW, BitTencourt-Oliveira MC AND Moura AN. 2010. Spatial-temporal variation in coiled and straight morphotypes of Cylindrospermopsis raciborskii (Wolsz) Seenayya et Subba Raju (Cyanobacteria). Acta Bot Bras 24: 585-591.

Dantas EW, BitTencourt-Oliveira MC AND Moura AN. 2012. Dynamics of phytoplankton associations in three reservoirs in northeastern Brazil assessed using Reynolds theory. Limnologica 42: 72-80.

DANTAS EW, Moura AN AND BitTEnCOURT-Oliveira MC. 2011. Cyanobacterial blooms in stratified and destratified eutrophic reservoirs in semi-arid region of Brazil. An Acad Bras Cienc 83: 1327-1338.

Ding W-X, Shen H-M, ZhU H-G, LeE N-L AND ONG C-N. 1999. Genotoxicity of Microcystic cyanobacteria extract of a water source in China. Mutat Res 442: 69-77.

Domingos P, RUBim TK, Molica RJR, AzEVEdo SMFO AND CARMICHAEL WW. 1999. First report of microcystin production by picoplanktonic cyanobacteria isolated from a northeast Brazilian drinking water supply. Environ Toxicol 14: 31-35.

FALCONER IR. 1991. Tumor promotion and liver injury caused by oral consumption of Cyanobacteria. Environ Toxicol Water Qual 6: 177-184.

FALCONER IR. 1996. Potential impact on human health of toxic cyanobacteria. Phycologia 35: 6-11. 
FALCONER IR AND BUCKLEY TH. 1989. Tumors promotion by Microcystis sp., a blue-green alga occurring in water supplies. Med J Aust 150: 351-352.

FALCONER IR AND HUMPAGE AR. 2001. Preliminary evidence for in vivo tumour initiation by oral administration of extracts of the blue-green alga Cylindrospermopsis raciborskii containing the toxin cylindrospermopsin. Environ Toxicol 16: 192-195.

FALCONER LR, HARDY SJ, HUMPAGE AR, FROSCIO SM, TOZER GJ AND HAWKINS PR. 1999. Hepatic and renal toxicity of the bluegreen alga (Cyanobacterium) Cylindrospermopsis raciborskii in a male swiss albino mice. Environ Toxicol 14: 143-150.

FAstner J, HeInZE R, HuMPAGE AR, MischKe U, EAGLEShaM GK AND CHORUS I. 2003. Cylindrospermopsis occurrence in two German lakes and preliminary assessment of toxicity and toxin production of Cylindrospermopsis raciborskii (Cyanobacteria) isolates. Toxicon 42: 313-321.

FORSBERG CAND RYDING SO. 1980. Eutrophication parameters and trophic state indices in 30 Swedish waste-receiving lakes. Arch Hydrobiol 89: 189-207.

Froscio SM, Humpage AR, Wickramasinghe W, SHaW G AND FALCONER IR. 2008. Interaction of the cyanobacterial toxin cylindrospermopsin with the eukaryotic protein synthesis system. Toxicon 51: 191-198.

HARADA K-I, OHTAMI I, IWAMOTO K, SUZYUKi M, WATANABE MF, WATANABE M AND TERAO K. 1994. Isolation of cylindrospermopsin from a cyanobacterium Umezakia natan and its screening method. Toxicon 32: 73-84.

HAWKINS PR AND GRIFFITHS DJ. 1993. Artificial destratification of a small tropical reservoir: effects upon the phytoplankton. Hydrobiologia 254: 169-181.

HAWKINS PR, RUNNEGAR MTC, JACKSON ARB AND FALCONER IR. 1985. Severe hepatotoxicity caused by the tropical cyanobacterium (Blue-Green Alga) Cylindrospermopsis raciborskii (Woloszynska) Seenaya et Subba Raju isolated from a domestic water supply reservoir. Appl Environ Microbiol 50: 1292-1295.

Hillebrand H, DÜRSElen C, Kirschtel D, Pollingher U AND ZOHARY T. 1999. Biovolume calculation for pelagic and benthic microalgae. J Phycol 35: 403-424.

Hooser SB, Beasley VR, Waite LL, KuKLENSChMidT MS, CARMICHAEL WW AND HASCHEK WM. 1991. Actin filament alterations in rat hepatocytes induced in vivo and in vitro by microcystin-LR, a hepatotoxin from the blue-green algae Microcystis aeruginosa. Vet Pathol 28: 259-266.

HUDNELL KH, DORTCH Q AND ZENICK H. 2008. An overview of the interagency, internation symposium on cyanobacterial harmful algal blooms (ISOC-HAB): advancing the scientific understanding of freshwater harmful algal blooms. In: HUDNELL KH (Ed), Cyanobacterial harmful algal blooms: State of the Science and Research Needs. Adv Exp Med Biol 619(XXIV): 1-16.

ITO E, Kondo F, TERAo K AND Harada K-I. 1997. Neoplastic nodular formation in mouse liver induced by repeated intraperitoneal injections of microcystin-LR. Toxicon 35: 1453-1457.
JOCHIMSEN EM ET AL. 1998. Liver failure and death after exposure to microcystin at a hemodialysis center in Brazil. N Engl J Med 338: 873-878.

LiR, CARMichael WW, BritTAin S, EAGLESHAM GK, SHaW GR, MAHAKHANT A, NOPARATNARAPORN N, YONGMANITCHAI W, KAYA K AND WATANABE MM. 2001. Isolation and identification of the cyanotoxin cylindrospermopsin and deoxy-cylindrospermopsin from a Thailand strain of Cylindrospermopsis raciborskii (Cyanobacteria). Toxicon 39: 973-980.

Lira GAST, ARAúJo EL, BitTenCOURT-Oliveira MC AND MOURA AN. 2011. Phytoplankton abundance, dominance and coexistence in an eutrophic reservoir in the state of Pernambuco, Northeast Brazil. An Acad Bras Cienc 83: 1313-1326.

LoBo E AND LEIGHTON G. 1986. Estructuras comunitarias de las fitocenosis planctonicas de los sistemas de Desembocaduras de rios y esteros de la zona central de Chile. Rev Biol Mar 22: 1-29.

MacKintosh C, BeAtTie KA, KLump S, COHEN P AND CodD GA. 1990. Cyanobacterial microcystin-LR is a potent and specific inhibitor of protein phosphatases 1 and 2A from both mammalians and higher plants. FEBS Lett 264: 187-192.

Mohamed ZA AND AL-SheHri AM. 2013. Assessment of cylindrospermopsin toxin in an arid Saudi lake containing dense cyanobacterial bloom. Environ Monit Assess 185: 2157-2166.

Molica RJR, Oliveira EJA, CARVAlho PVVC, Costa ANSF, Cunha MCC, Melo GL and AzEvedo SMFO. 2005. Occurrence of saxitoxins and an anatoxin-a(s)-like anticholinesterase in a Brazilian drinking water supply. Harmful Algae 4: 743-753.

Moschini-CARlos V, Bortoli S, PINTO E, Nishimura PY, Freitas LG, POMPÊO MLM AND DÖRR F. 2009. Cyanobacteria and cyanotoxin in the Billings reservoir (São Paulo, SP, Brazil). Limnetica 28: 273-282.

Moura AN, Dantas EW, Oliveira HSB AND BitTENCOURTOLIVEIRA MC. 2011. Vertical and temporal dynamics of cyanobacteria in the Carpina potable water reservoir in Northeastern Brazil. Braz J Biol 71: 451-459.

New Zealand, Ministry of Health. 2008. Drinkingwater Standards for New Zealand 2005 (Revised 2008). Wellington: Ministry of Health, 163 p.

NHMRC, NRMMC. 2011. Australian Drinking Water Guidelines Paper 6 National Water Quality Management Strategy. National Health and Medical Research Council, National Resource Management Ministerial Council, Common wealth of Australia, Canberra.

NiSHIWAKI-MATSUSHIMA R, OHTA T, NiSHIWAKI S, SUGUNUMA M, KOHYAMA K, ISHIKAWA T, CARMICHAEL WW AND FUJIKI H. 1992. Liver tumor promotion by the cyanobacterial cyclic peptide toxin microcystin-LR. J Cancer Res Clin Oncol 118: 420-424.

O'NeIL J AND DENNISON WC. 2005. Lyngbya majuscule in Southeast Queensland. In: ABAL E and DENNISON WC (Eds), Healthy Waterways - Healthy catchments. Brisbane: South East Queensland Reginal Water Quality Strategy, Brisbane City Council, Australia, p.119-148. 
O'NeIL JM, DAVIS TW, Burford MA AND GOBLER CJ. 2012. The rise of harmful cyanobacteria blooms: The potential roles of eutrophication and climate change. Harmful Algae 14: 313-334.

OHtani I, Moore RE And Runnegar MTC. 1992. Cylindrospermopsin, a potent hepatotoxin from the blue-greenalga Cylindrospermopsis raciborskii. J Am Chem Soc 114: 7941-7942.

PAERL HW, HALl NS AND CALANDRINO ES. 2011. Controlling harmful cyanobacterial blooms in a world experiencing anthropogenic and climatic-induced change. Sci Total Environ 409: 1739-1745.

PAERL HW AND HUISMAN J. 2009. Climate change: a catalyst for global expansion of harmful cyanobacterial blooms. Eviron Microb Rep 1: 27-37.

PaUl VJ, Thacker R, BANKS K AND GOLUBiC S. 2005. Benthic cyanobacterial bloom impacts on the reefs of South Florida (Broward County, USA). Coral Reefs 24: 693-697.

Piccin-SAntos V AND BitTencourt-Oliveira MC. 2012 Toxic cyanobacteria in four Brazilian water supply reservoirs. J Environ Prot 3: 68-73.

Preussel K, Stuken A, Wiedner C, Chorus I And Fastner J. 2006. First report on cylindrospermopsin producing Aphanizomenon flos-aquae (Cyanobacteria) isolated from two German lakes. Toxicon 47: 156-162.

Richardson LL, SeKar R, Myers JL, Gantar M, Voss JD, KACZMARSKY L, REMILY ER, BOYER GL AND ZIMBA PV. 2007. The presence of the cyanobacterial toxin microcystin in black band disease of corals. FEMS Microbiol Lett 272: 182-187.

SAKer ML, Nogueira ICG, VAsconcelos VM, NeIlan BA, EAglesham GK AND Pereira P. 2003. First report and toxicological assessment of the cyanobacterium Cylindrospermopsis raciborskii from Portuguese freshwaters. Ecotoxicol Environ Saf 55: 243-250.

SANT'ANNA CL, GAMA-JR WA, AZEVEDO MTP AND KOMÁREK J. 2011. New morphospecies of Chamaesiphon (Cyanobacteria) from Atlantic rainforest, Brazil. Fottea 11: 25-30.

SCHEMBRI MA, NEILAN BA AND SAINT CP. 2001. Identification of genes implicated in toxin production in the cyanobacterium Cylindrospermopsis raciborskii. Environ Toxicol 16: 413-421.

SEAWRIGHT AA, NOLAN CC, SHAW GR, CHISWELl RK, NORRIS RL, MOORE MR AND SMITH MJ. 1999. The oral toxicity for mice of the tropical cyanobacterium Cylindrospermopsis raciborskii (Woloszynska). Environ Toxicol 14: 135-142.

SeIFERT M, MCGregor G, EAgLesham G, Wickramasinghe W AND SHAW G. 2007. First evidence for the production of cylindrospermopsin and deoxy-cylindrospermopsin by the freshwater benthic cyanobacterium, Lyngbya wollei (Farlow ex Gornont) Speziale and Dyck. Harmful Algae 6: 73-80.

SMITH FMJ, WoOd SA, VAN GINKEL R, BROADY PA AND GAW S. 2011. First report of saxitoxin production by a species of the freshwater benthic cyanobacterium, Scytonema Agardh. Toxicon 57: 566-573.
Smith FMJ, Wood SA, Wilks T, Kelly D, BroAdy PA, WiLliamson W AND GAW S. 2012. Survey of Scytonema (Cyanobacteria) and associated saxitoxins in the littoral zone of recreational lakes in Canterbury, New Zealand. Phycologia 51: 542-551.

SoAres RM, CAGIDO VR, FERraro RB, MEYER-FERnandes JR, ROCCO PRM, ZIN WA AND AZEVEDO SMFO. 2007. Effects of microcystin-LR on mouse lungs. Toxicon 50: 330-338.

SoARes RM, Yuan M, Servaites JC, Delgado A, Magalhães VF, Hilborn ED, CARMichael WW AND AZEVEDO SMFO. 2006. Sub-lethal exposure from microcystins to renal insufficiency patients in Rio de Janeiro-Brazil. Environ Toxicol 2: 95-103.

Sotero-Santos RB, CARVAlho GE, Dellamano-Oliveira MJ AND RochA O. 2008. Occurrence and toxicity of an Anabaena bloom in a Tropical reservoir (Southeast Brazil). Harmful Algae 7: 590-598.

Sotero-Santos RB, Silva CRSE, Verani NF, NonaKa KO AND RochA O. 2006. Toxicity of a cyanobacteria bloom in Barra Bonita reservoir (Middle Tietê River, São Paulo, Brazil). Ecotoxicol Environ Saf 64: 163-170.

SPOOF L, BerG KA, RAPALA J, LAHTI K, LEPISTO L, METCALF JS, CodD GA AND MERILUOTO J. 2006. First observation of cylindrospermopsin in Anabaena lapponica isolated from the boreal environment (Finland). Environ Toxicol 21: $552-560$

STIRLING DJ AND QUILLIAM MA. 2001. First report of the cyanobacterial toxin cylindrospermopsin in New Zealand. Toxicon 39: 1219-1222.

Suganuma M, FuJiki H, Suguri H, Yoshizawa S, Hirota M, NAKAYASU M, OJIKA M, WAKAMATSU K, YAMADA K AND SUGIMURA T. 1988. Okadaic acid, an additional non-phorbol-12-tetradecanoate-13-acetate-type tumor promoter. Proc Natl Acad Sci USA 85: 1768-1771.

TeIXeIra MGLC, Costa MCN, CARVALho VLP, PEREIRA MS AND HaGe E. 1993. Gastroenteritis epidemic in the area of the Itaparica, Bahia, Brazil. Bull Pan Am Health Organ 27: $244-253$

TERAO K, OHMORI S, IgARASHI K, OHTANI I, WATANABE MF, HARADA K-I, ITO E AND WATANABE MF. 1994. Electron microscopic studies on experimental poisoning in mice induced by cylindrospermopsin isolated from blue-green alga Umezakia natans. Toxicon 32: 833-843.

Ueno Y, Nagata S, Tsutsumi T, Hasegawa A, Watanabe MF, PARK HD, ChEN G-C, CHEN G AND YU S-Z. 1996. Detection of microcystins, a blue-green algal hepatotoxin, in drinking water sampled in Haimen and Fusui, endemic areas of primary liver cancer in China, by highly sensitive immunoassay. Carcinogenesis 17: 1317-1321.

UTERMÖHL H. 1958. Zur Vervollkommung der quantitativen phytoplanktonmethodik. Int Ver Theor Angew Limnol Verh 9: 1-38.

WETZEL RG AND LIKENS GE. 2000. Limnological analyses. New York: Springer Verlag, $3^{\text {rd }}$ ed., 429 p. 
Yilmaz M, Philips EJ, SZABo NJ AND BADYlaK S. 2008. A comparative study of Florida strains of Cylindrospermopsis and Aphanizomenon for cylindrospermopsin production. Toxicon 51: 130-139.

ZapomĚlová E, Jezberová J, HrouzeK P, Hisem D, REHÁKOVÁ K AND KomÁRKOVÁ J. 2009. Polyphasic characterization of three strains of Anabaena reniformis and Aphanizomenon aphanizomenoides (cyanobacteria) and their reclassification to Sphaerospermum gen. nov. (incl. Anabaena kisseleviana). J Phycol 45: 1363-1373.
ZAPOMĚlovÁ E, JEZBEROVÁ J, HROUZEK P, HISEM D, REHÁKOVÁ K AND KomÁRKOVÁ J. 2010. Polyphasic characterization of three strains of Anabaena reniformis and Aphanizomenon aphanizomenoides (cyanobacteria) and their reclassification to Sphaerospermum Gen. Nov. (incl. Anabaena kisseleviana). J Phycol 46: 415.

ZHOU L, YU H AND CHEN K. 2002. Relationship between microcystin in drinking water and colorectal cancer. Biomed Environ Sci 15: 166-171. 